\title{
FEASIBILITY STUDY BUILDING MATERIAL OF SOIL PEAT CENTRAL KALIMANTAN
}

\author{
Aventi, Ir. MT \\ Madya Researcher - Research Institute For Human Settlements - Public Works Ministry \\ Jalan Panyaungan - Cileunyi Wetan - Kabupaten Bandung - Indonesia
}

\begin{abstract}
I have done research about using soil peat from Central Kalimantan as local building material in making red brick and tile ceramic, conclude : (a) Potency building material from mining material group $\mathrm{C}$ shaped soil peat generally stock enough and from 6 (six) sample that tested in laboratory as red brick and ceramic tile, fulfill technical requirements based on characeristics ; (b) Potency building material from mining material group $\mathrm{C}$ shaped quartz sand stock enough and can utilized as mortar plester ; (c) Location has choosen unit production red brick and ceramic tile that can developed technical ways is Lamunti, Palem Bahem, and Pulau Telo ; (d) Potency building material shaped soil peat stock enough and can utilized as red brick and tile ceramic, still need to do trial test production in field, that is means society around can spare transfer technology so hopefully can grow and develop new industries ; and (e) Utilization building material sand as building material, need to do testing in laboratory first before used.
\end{abstract}

This research is to continue that research result, by calculate how much the cost was needed to produce red brick and tile ceramic from soil peat Central Kalimantan, that cost will be cheaper or more expensive, compare with the cost of red brick and tile ceramic that have been sold in market.

This research also to calculate feasibility study if that red brick and tile ceramic from soil peat Central Kalimantan was produced by mass.

Keywords: feasibility study, red brick, tile ceramic.

\section{INTRODUCTION}

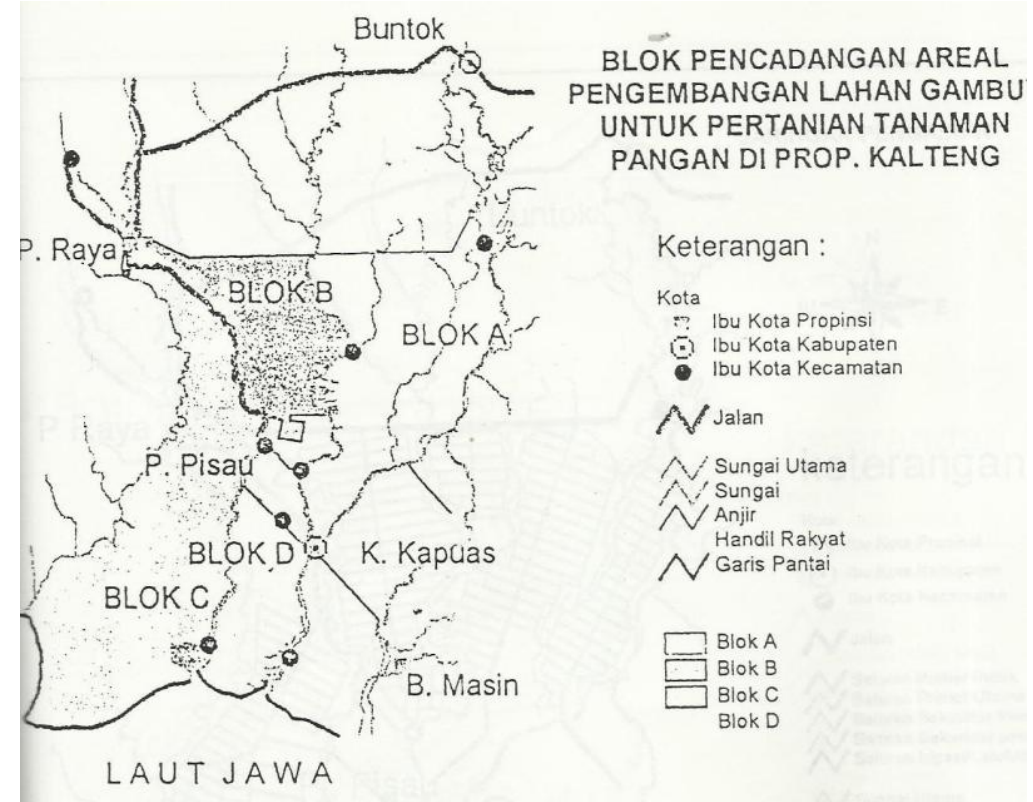

Corresponding Author: aventi_kusuma@yahoo.co.id or aventi.kusuma@gmail.com 
Province Central Kalimantan have wide area $153.564 \mathrm{~km}^{2}$ with geography position between 0' $45^{\circ}$ North Latitude and 3' $30^{\circ}$ South Latitude and between $111^{\circ}-115^{\circ}$ East Longitude contains 14 district and 1 sub district/city. Specifically, Project Development Peat Area 1 million hectare area block A and B is in District Kapuas $\left(38.400 \mathrm{~km}^{2}\right)$, District Pulang Pisau (8.997 $\left.\mathrm{km}^{2}\right)$ and District Barito Selatan $\left(12.664 \mathrm{~km}^{2}\right)$.

Any location taken sample clay that possible can developed technical or economical way :

Transmigration settlements SP-2 - SKP-A, sub district Dadahup (area A), that is towards headwaters Kapuas river, sampling soil peat $\pm 10 \mathrm{~m}$ from river-bank with depth $\pm 1 \mathrm{~m}$ above land level, transportation can go through as long as 2 hours from Kapuas city by speed boat.

Sub district Lamunti (area B), that is towards headwaters Kapuas river. Sampling soil peat \pm 50 $\mathrm{m}$ from river-bank with depth $\pm 1 \mathrm{~m}$ under soil peat level, can go through as long as 2 hours from Kapuas by speed boat.

Village Palem Bahem (area D), Village Pangkuh sub district Pandihbatu, that is towards downstream Kapuas river also sampling soil peat $\pm 50 \mathrm{~m}$ from river-bank with depth $\pm 1 \mathrm{~m}$ under soil peat level. Time to go to that location as long as 3 hours from Kapuas regency by speed boat.

Village Buntoi, village Pulang Pisau - sub district Kahayan Hilir, that is towards downstream Kapuas river. Sampling soil peat $\pm 150 \mathrm{~m}$ from river-bank with depth $\pm 1 \mathrm{~m}$, time to go to the location need 1,5 hours from Kapuas by speed boat.

Sub district Kahayan Hilir - Pulang Pisau (area F) - village Gohong - km 7 from Pulang Pisau, sampling $\pm 400 \mathrm{~m}$ from bridge Palangkaraya.

Village Kahayan also taken 2 sample soil peat with kinds black soil peat and reddish white soil peat.

Potency sand generally be found at stream flow Kapuas river. Sampling sand from location mining in stream flow river be located in village Keladan, sub district Mentangai. Mining method sand that be in the bottom of the river sucked by pump at depth $\pm 6 \mathrm{~m}$. Capacity production mining $\pm 60 \mathrm{~m}^{3} /$ day by boat.

To go to location mining need time \pm 2 hours from Kapuas regency by speed boat. Result mining sand carried to Kapuas city.

The sand used as mortar, concrete, conblock, paving block, etc.

Location exploition Barito river be in a place 100 $m$ from side of the highway - village Hilir Sper village Kampel - South barito. In 1 day produce 1 $1 / 2 \mathrm{~m}^{3}$ sand. Before sold the sand gradation done sieve using sieve $0,5 \mathrm{~cm}$.

\section{METHODOLOGY}

This research is to calculate feasibility study red brick and tile ceramic from soil peat Central Kalimantan was produced by mass.

\section{RESEARCH RESULTS}

Only soil peat from Pulau Telo that have $\mathrm{SiO}_{2}$ content $51.79 \%$, include category clay. Soil peat from other location still under limit content $\mathrm{SiO}_{2}$ that required for clay. But the value almost same, means soil peat from other location also have chemical characteristic closer to clay.

Soil peat from six location have chemical content $\mathrm{Al}_{2} \mathrm{O}_{3}, \mathrm{Fe}_{2} \mathrm{O}_{3}, \mathrm{CaO}, \mathrm{MgO}$ same with chemical content of clay.

Soil peat from Palem Bahem and Pulau Telo have $\mathrm{SO}_{3}$ content 0 , same with chemical content clay. Soil peat from Dadahup, Lamunti, Handel Usang, and Buntoi have $\mathrm{SO}_{3}$ content above $\mathrm{SO}_{3}$ content of clay.All sample soil have dry shrinkage value between $8 \%$ - $11 \%$, so include category soil very sensitive to drying, so need special treatment on process drying after produce by no crack and no break of production results.

Similarly from test results on sensitivity value to drying /DSE 3 sample have DSE value between 1 -2 and 3 sample soil peat have DSE value above 2 , than sample soil taken in general can categorized sensitive to drying. 
Test results to index plasticity value shows that 2 sample have IP value between $20 \%-30 \%$ and 4 sample soil above $30 \%$, than the sample soil include plastis and very plastis loam.

Based on analysis large grain was described in diagram Winkler, generally the sample soil can be described as material ceramic tile and red brick.

While based on flexure strength dry condition whole sample soil have flexure strength above 10 $\mathrm{kg} / \mathrm{cm}^{2}$ so generally have base strength good enough.

Next done fire test with various temperature, that is $800^{\circ} \mathrm{C}, 850^{\circ} \mathrm{C}$, and $900^{\circ} \mathrm{C}$ to know temperature maximum or burning temperature was needed so obtained best results by flexure strength was obtained from sample test after burned.

From the testing founded optimal burning temperature, that is $900^{\circ} \mathrm{C}$ with result optimal flexure strength and fulfill technical requirements of sample test to all location.

From sample soil peat was taken from the field, next doing test to make tile ceramic and red brick to know technical properties as base its development.

From testing result of soil peat, obtained data :

Shape tile ceramic : curve flat type M-20 ;

Table 1. Nominal size tile ceramic

\begin{tabular}{|l|c|c|c|}
\hline \multicolumn{1}{|c|}{ Size } & Total & Utilized & Hook \\
\hline Length, cm & 32,2 & 24,2 & 3,2 \\
\hline Width, cm & 23,5 & 20,0 & 1,5 \\
\hline Wide, cm & 1,45 & - & 0,8 \\
\hline Weight, kg & 2,5 & - & - \\
\hline
\end{tabular}

Water absorption : not absorp.

Tile curve flat is tile with cross section middle part flat and the sides curved.

Tile ceramic for whole quality level must resistance to water absorption. At testing water absorption, water can not dripping from bottom part of tile in less than 2 hours.
Tile ceramic of soil peat resistance to water absorption or not absorp water, because at the time testing water absorption, water not dripping from bottom part of tile in less than 2 hours.

Requirements size of tile ceramic according to SNI 03-2095-1991 "Quality and test method tile ceramic":

Table 2. Size of tile ceramic

\begin{tabular}{|c|l|c|c|c|c|}
\hline \multirow{2}{*}{ No. } & \multicolumn{1}{|c|}{ Description } & \multicolumn{3}{|c|}{ Tile Ceramic } & \multirow{2}{*}{ Explanation } \\
\cline { 3 - 5 } & & Small & Medium & Large & \\
\hline 2 & $\begin{array}{l}\text { Length utilized } \\
\text { (reng distance, mm) }\end{array}$ & 200 & 250 & 333 & Penyimpangan \\
\hline 3 & Width utilized, mm & 200 & 200 & 200 & \\
\hline 4 & $\begin{array}{l}\text { Distance cover } \\
\text { longitudinal, min, } \\
\text { mm }\end{array}$ & 40 & 50 & 67 & \\
\hline 5 & $\begin{array}{l}\text { Distance cover } \\
\text { transverse, min, } \\
\text { mm }\end{array}$ & 40 & 40 & 40 & \\
\hline $\begin{array}{l}\text { Hooks : } \\
\text { Height, mm }\end{array}$ & 10 & 10 & 10 & \\
Length, mm & 30 & 30 & & \\
\hline
\end{tabular}




\begin{tabular}{|l|l|l|l|l|l|}
\hline \multirow{2}{*}{ No. } & Description & \multicolumn{3}{|c|}{ Tile Ceramic } & \multirow{2}{*}{ Explanation } \\
\cline { 3 - 5 } & & Small & Medium & Large & \\
\hline & Width, $\mathrm{mm}$ & & & & \\
\hline
\end{tabular}

Tile ceramic of soil peat have medium sized.

Shape red brick : rectangular (massive) with nominal size :

$\begin{array}{llll}\text { Length, } \mathrm{cm} & 19,4 \quad ; \quad \text { wide, } \mathrm{cm} \quad: 4,8\end{array}$

Width, $\mathrm{cm} \quad 9,7 \quad ; \quad$ weight, $\mathrm{kg} \quad: 1,5$

Table 3. Characteristics of red brick'

\begin{tabular}{|c|l|c|c|c|c|c|c|}
\hline No. & Location & $\begin{array}{c}\text { Water } \\
\text { Content } \\
\text { Average } \\
(\boldsymbol{\%})\end{array}$ & $\begin{array}{c}\text { Water } \\
\text { Absorption } \\
(\boldsymbol{\%})\end{array}$ & $\begin{array}{c}\text { Furnace } \\
\text { Absorption (gr } \\
\mathbf{/ d m}^{\mathbf{2}} / \mathbf{m i n u t e )}\end{array}$ & Density & $\begin{array}{c}\text { Pressure } \\
\text { Strength } \\
\text { Average } \\
\left(\mathbf{k g} / \mathbf{c m}^{\mathbf{2}}\right)\end{array}$ & $\begin{array}{c}\text { Salt } \\
\text { Conten } \\
\mathbf{t}\end{array}$ \\
\hline 1 & Lamunti & 0,34 & 19,16 & 58 & 1,68 & 27,62 & No \\
\hline 2 & $\begin{array}{l}\text { Palem } \\
\text { Bahem }\end{array}$ & 0,48 & 17,96 & 50 & 1,70 & 23,27 & No \\
\hline 3 & P. Telo & 0,34 & 18,51 & 42 & 1,68 & - & No \\
\hline
\end{tabular}

Outside view red brick of soil peat must have sides that sharp and angle, the field of flat side not indicate any crack and changing the form of excessive.

Red brick size according to SNI 15-2094-1991 $:^{7)}$

Length : maximal $240 \mathrm{~mm}$ and minimal $230 \mathrm{~mm}$.

Width : maximal $15 \mathrm{~mm}$ and minimal $110 \mathrm{~mm}$.

Height : maximal $52 \mathrm{~mm}$ and minimal $50 \mathrm{~mm}$.

Pressure strength red brick must fulfill requirements according to SNI 15-2094-1991 "Quality and test method red brick massive" :

Quality Level Average Pressure Strength $\left(\mathrm{kg} / \mathrm{cm}^{2}\right)$

$$
\begin{array}{cc}
\text { I } & >100 \\
\text { II } & 80-100 \\
\text { III } & 60-80
\end{array}
$$

Red brick of soil peat have average pressure strength very low, not fulfill requirements SNI 152094-1991, under average pressure strength quality III. To increase average pressure strength red brick of soil peat, must go through combustion process beforehand.
Salt content can not show signs that according test result represented hazard, that is if the red brick soaked in water with standing position half soak, for at least 3 days, has seems white spots on the surface.

If less than $50 \%$ called not hazard, but if more than $50 \%$ hazard.

Red brick of soil peat does not contain salt content, so not hazard.

\section{ANALYSIS AND DISCUSSION}

Machine was used in making red brick and tile ceramic : 


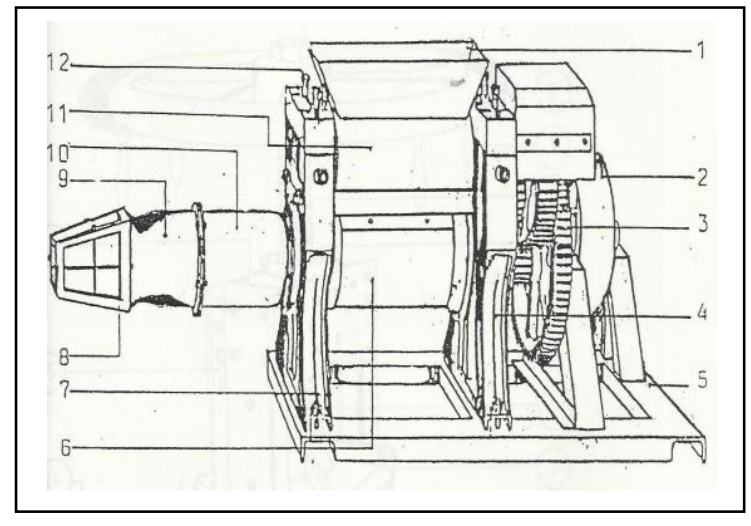

Extruder, machine to make red brick

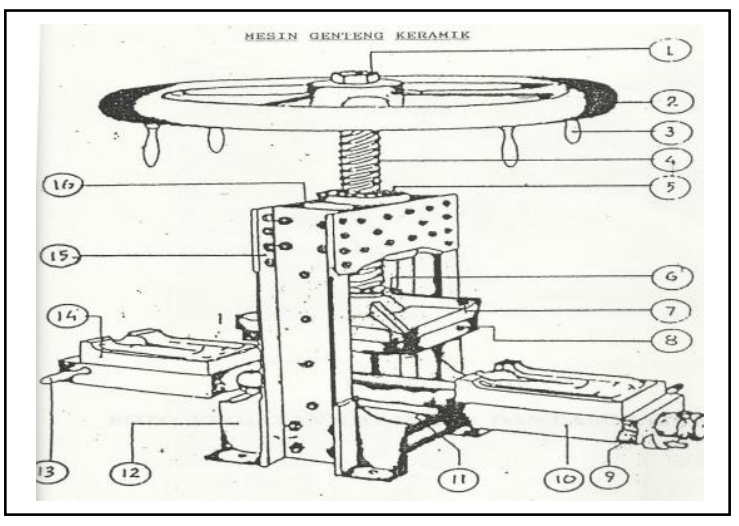

Machine to make tile ceramic

\section{ESTIMATION INVESTATION AND COST PRODUCTION RED BRICK}

List Price Unit Tools Production Red Brick

Capacity :

$\begin{array}{ll}288.000 & \text { pieces/year } \\ 960 & \text { pieces/day }\end{array}$

\begin{tabular}{|c|c|c|c|c|c|}
\hline \multirow[b]{2}{*}{ No. } & \multirow[b]{2}{*}{ Kind of Tools } & \multirow[b]{2}{*}{ Quantity } & \multirow[b]{2}{*}{ Unity } & \multicolumn{2}{|c|}{ Price Rp. } \\
\hline & & & & Unit & Total \\
\hline 1 & Extruder machine & 2 & Unit & $20.000 .000,00$ & $40.000 .000,00$ \\
\hline 2 & Mixer machine & 1 & Unit & $20.000 .000,00$ & $20.000 .000,00$ \\
\hline 3 & Tool assist & 1 & Unit & $5.000 .000,00$ & $5.000 .000,00$ \\
\hline \multicolumn{5}{|r|}{ Total } & $65.000 .000,00$ \\
\hline & \multicolumn{3}{|c|}{ Total Machines and Tools } & $=\mathbf{R p}$. & $65.000 .000,00$ \\
\hline & \multicolumn{3}{|c|}{ Depreciation per year } & $=\mathbf{R p}$. & $6.500 .000,00$ \\
\hline & \multicolumn{3}{|c|}{ Depreciation per piece product } & $=\mathbf{R p}$. & 22,57 \\
\hline
\end{tabular}

Investation Unit Production Red Brick

\begin{tabular}{|c|l|r|}
\multicolumn{1}{|c|}{$\mathbf{2 8 8 . 0 0 0}$ pieces/year } \\
\hline No. & \multicolumn{1}{|c|}{ COST OF INVESTATION } & \multicolumn{1}{c|}{$\begin{array}{c}\text { PRICE } \\
\text { (Rp.) }\end{array}$} \\
\hline 1 & TOOLS PRODUCTION RED BRICK & $65.000 .000,00$ \\
2 & COST INSTALLATION ELECTRICITY AND WATER & $5.000 .000,00$ \\
3 & LAND FACTORY \& CONCESSION (250 m2) & $125.000 .000,00$ \\
4 & BUILDING PRODUCTION (125 m2) & $125.000 .000,00$ \\
5 & EQUITY WORK 3 MONTHS & $54.000,00$ \\
\hline & TOTAL INVESTATION & $\mathbf{3 2 0 . 0 5 4 . 0 0 0 , 0 0}$ \\
\hline
\end{tabular}

Source Payment

Credit bank

$75 \% \quad 240.040 .500,00$ 


Self equity

$25 \% \quad 80.013 .500,00$

T O T A L

320.054.000,00

\section{Estimation Cost Production Red Brick}

Capacity : 288.000 pieces/year

Salary worker

\begin{tabular}{|c|l|l|c|l|}
\hline No. & Explanation & Salary/month $(\mathbf{R p})$ & Worker & Total Salary $(\mathbf{R p )})$ \\
$\mathbf{1}$ & Direct Salary & & & \\
& Worker & $1.700 .000,00$ & 3 & $5.100 .000,00$ \\
& Assistance & $1.500 .000,00$ & 3 & $4.500 .000,00$ \\
\hline
\end{tabular}

\section{Direct Salary}

Salary/month

Rp. $\quad 9.600 .000,00$

Salary/year

Rp. 115.200.000,00

Allowance 1 Month Salary Rp. $\quad \mathbf{9 . 6 0 0 . 0 0 0 , 0 0}$

Total Salary \& Allowance Rp. 124.800.000,00

Salary \& Allowance/piece Rp.

433,33

\section{Estimation Cost Production Red Brick}

Capacity Production : $\mathbf{9 . 6 0 0}$ pieces/10 days

Mixture composition $1: 3$

Day work : $\mathbf{3 0 0}$ days/year

Capacity per year : $\mathbf{2 8 8 . 0 0 0}$ pieces/year

Direct Cost :

\begin{tabular}{|c|c|c|c|c|}
\hline Explanation & Unity & Volume & $\begin{array}{l}\text { Unit Price } \\
\qquad \text { (Rp) }\end{array}$ & $\begin{array}{c}\text { Total Price } \\
\text { (Rp) }\end{array}$ \\
\hline Solar and Olie & Ltr & 288 & $6.700,00$ & $1.929 .600,00$ \\
\hline Sand & $\mathrm{Gr}$ & 151.200 .000 & 0,75 & $113.400 .000,00$ \\
\hline Clay peat & $\mathrm{Gr}$ & 550.800 .000 & 0,50 & $275.400 .000,00$ \\
\hline Electricity & Kwh & 2.400 & $4.200,00$ & $10.080 .000,00$ \\
\hline Material and energy & & & & $400.809 .600,00$ \\
\hline Salary & & & & - \\
\hline
\end{tabular}

T o t a l $\quad 400.809 .600,00$

Indirect Cost :
\begin{tabular}{|l|c|c|c|c|}
\hline \multicolumn{1}{|c|}{ Explanation } & Unity & Volume & $\begin{array}{c}\text { Unit Price } \\
(\mathbf{R p})\end{array}$ & $\begin{array}{c}\text { Total Price } \\
(\mathbf{R p})\end{array}$ \\
\hline Depreciation & $\%$ & 5 & $65.000 .000,00$ & $3.250 .000,00$ \\
Overhead & $\%$ & 5 & & -
\end{tabular}




\begin{tabular}{|l|c|c|r|r|}
\hline Maintenance & Ls & 0,05 & $65.000 .000,00$ & $3.250 .000,00$ \\
\hline Salary & \multicolumn{4}{|c|}{-} \\
\hline & \multicolumn{4}{|c|}{ T o t a l 6.500.000,00 } \\
\hline
\end{tabular}

Payment + Equity Bank per year $\quad \mathbf{1 9 0 . 6 7 7 . 0 0 4 , 2 6}$

TOTAL $(1+2+3) \quad 597.986 .604,26$

MAIN PRICE PER PIECE $\quad \mathbf{2 . 0 7 6 , 3 4}$

Profit $20 \% \mathbf{4 1 5 , 2 7}$

Taxes $10 \%$ 207,63

SELL PRICE PER PIECE 2.699,25

Break Event Point (BEP)

Unit Production Red Brick Capacity : $\quad 9.600$ pieces/10 days

288.000 pieces/year

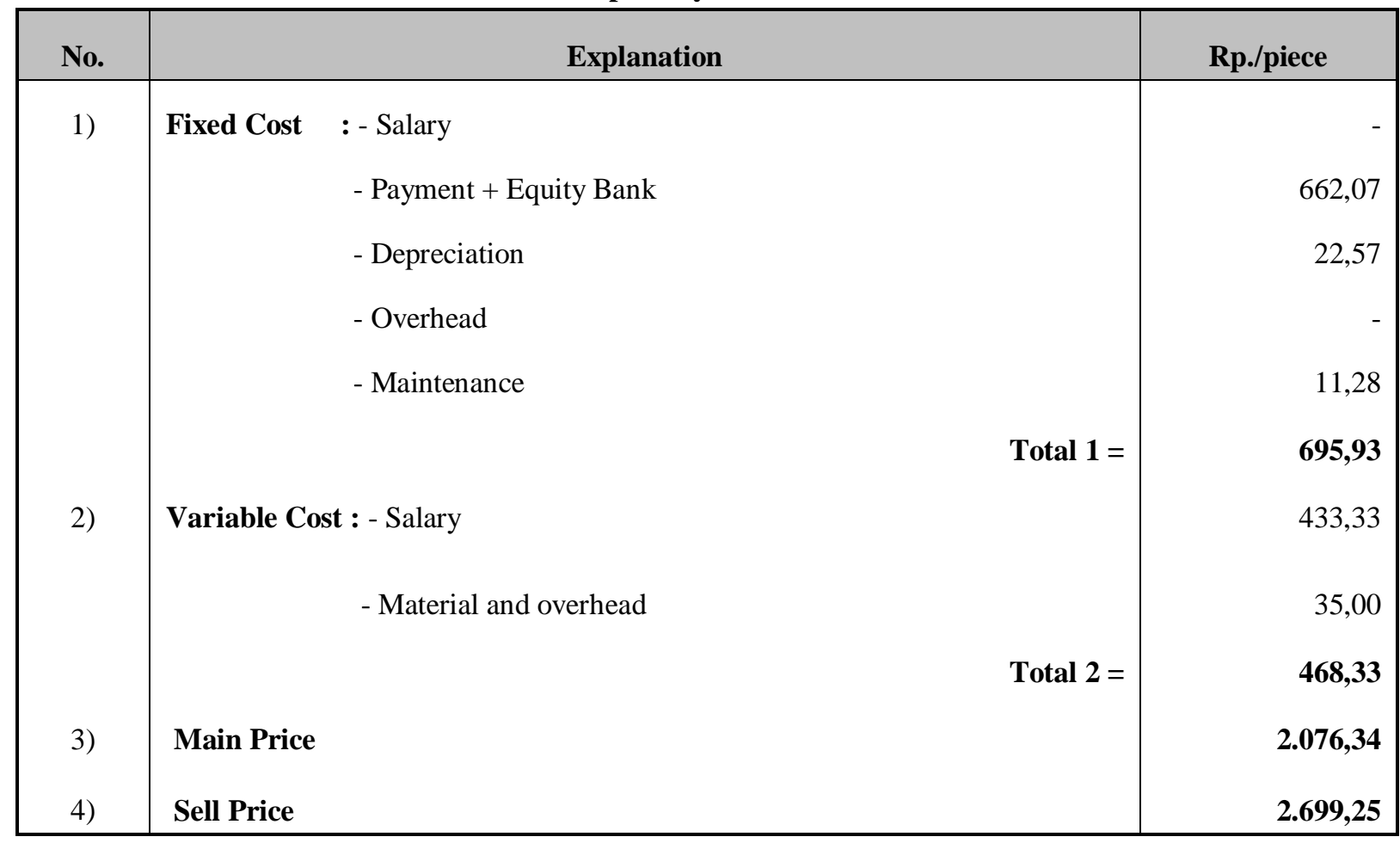

Fixed Cost

BEP = ------------- X $100 \%=\mathbf{3 1 , 1 9 \%}$

Sell price - Var. Cost 
Cash flow unit production red brick

\begin{tabular}{|c|c|c|c|c|c|c|c|c|c|c|c|}
\hline No. & Explanation & 2016 & 2017 & 2018 & 2019 & 2020 & 2021 & 2022 & 2023 & 2024 & 2025 \\
\hline 1 & Balance cash beginning & 0 & 0 & 179.395 .981 & 376.731 .561 & 593.800 .698 & 832.576 .749 & 1.095 .230 .405 & 1.384 .149 .427 & 1.701 .960 .351 & 2.051 .552 .367 \\
\hline 2 & Income & 320.054 .000 & 777.382 .586 & 855.120 .844 & 940.632 .928 & 1.034 .696 .221 & 1.138 .165 .843 & 1.251 .982 .428 & 1.377.180.671 & 1.514 .898 .738 & 1.666.388.611 \\
\hline 3 & Outcome & 320.054 .000 & 597.986 .604 & 657.785 .265 & 723.563 .791 & 795.920 .170 & 875.512 .187 & 963.063 .406 & 1.059 .369 .747 & 1.165 .306 .721 & 1.281 .837 .393 \\
\hline 4 & Difference cash (2-3) & 0 & 179.395 .981 & 197.335.579 & 217.069 .137 & 238.776 .051 & 262.653 .656 & 288.919 .022 & 317.810 .924 & 349.592 .016 & 384.551 .218 \\
\hline 5 & Balance cash ending & 0 & 179.395.981 & 376.731 .561 & 593.800 .698 & 832.576 .749 & 1.095 .230 .405 & 1.384.149.427 & 1.701 .960 .351 & 2.051.552.367 & 2.436 .103 .586 \\
\hline
\end{tabular}

Main price red brick Rp. 2.076,34/piece

Sell price red brick Rp. 2.699,25/piece

Capacity production $\mathbf{2 8 8 . 0 0 0}$ pieces/year

Cost production red brick estimation increase as much as : $\mathbf{1 0} \% /$ year

Total investation Rp. 320.054.000,- 


\section{Calculation Internal Rate of Return (IRR) Production Red Brick}

Capacity Prod.: $\mathbf{2 8 8 . 0 0 0}$ pieces/year

Sell Price : 2.699,25 rupiah/piece

Main Price : 2.076,34 rupiah/piece

Investation : 320.054.000,00 rupiah

\begin{tabular}{|l|r|r|r|r|r|c|}
\hline \multicolumn{1}{|c|}{ Year } & $\mathbf{0}$ & $\mathbf{1}$ & $\mathbf{2}$ & $\mathbf{3}$ & $\mathbf{4}$ & $\mathbf{5}$ \\
\hline Income & 0 & 777382586 & 777382585,5 & 777382586 & 777382586 & 777382585,5 \\
\hline Outcome & 320054000 & 597986604 & 597986604,3 & 597986604 & 597986604 & 597986604,3 \\
\hline Net Cash Flow : & -320054000 & 179395981 & 179395981,3 & 179395981 & 179395981 & 179395981,3 \\
\hline NPV 50 \% : & 1 & 0,6666667 & 0,444444444 & 0,2962963 & 0,19753086 & 0,131687243 \\
\hline NPV 40 \% : & 1 & 0,7142857 & 0,510204082 & 0,36443149 & 0,2603082 & 0,185934432 \\
\hline NPV 30\%: & 1 & 0,7692308 & 0,591715976 & 0,45516614 & 0,3501278 & 0,269329074 \\
\hline NPV 20\%: & 1 & 0,8333333 & 0,694444444 & 0,5787037 & 0,48225309 & 0,401877572 \\
\hline
\end{tabular}

Total NPV $50 \%$ : -8510361,732 rupiah

Total NPV $40 \%: \mathbf{4 5 . 0 4 6 . 2 2 8 , 4 5}$ rupiah

Total NPV $30 \%: \mathbf{1 1 6 . 8 7 7 . 4 2 5 , 6 6}$ rupiah

Total NPV $20 \%: \mathbf{2 1 6 . 4 4 9 . 7 9 9 , 4 6}$ rupiah

$\mathrm{IRR}=\mathbf{4 6 , 2 7} \%$

Pay Back Period $=\mathbf{1 , 7 8}$ year

Profit $=\mathbf{2 0 , 0 0 \%}$ 
Aventi, Ir. MT /Feasibility Study Building Material of Soil Peat Central Kalimantan

\begin{tabular}{|c|c|c|c|c|c|c|c|c|c|}
\hline No. & Explanation & 2016 & 2017 & 2018 & 2019 & 2020 & 2021 & 2022 & 2023 \\
\hline 1 & Income & 777.382 .586 & 855.120 .844 & 940.632 .928 & 1.034 .696 .221 & 1.138 .165 .843 & 1.251 .982 .428 & 1.377.180.671 & 1.514 .898 .738 \\
\hline 2 & Cost Production & 597.986 .604 & 657.785 .265 & 723.563 .791 & 795.920 .170 & 875.512 .187 & 963.063 .406 & 1.059 .369 .747 & 1.165 .306 .721 \\
\hline 3 & Profit before equity \& tax & 179.395 .981 & 197.335 .579 & 217.069 .137 & 238.776 .051 & 262.653 .656 & 288.919 .022 & 317.810 .924 & 349.592 .016 \\
\hline 4 & Equity loan + payment & 119.342 .552 & 119.342 .552 & 119.342 .552 & 119.342 .552 & 119.342 .552 & 0 & 0 & 0 \\
\hline 5 & Profit before tax & 60.053 .429 & 77.993 .027 & 97.726 .585 & 119.433 .499 & 143.311 .104 & 288.919 .022 & 317.810 .924 & 349.592 .016 \\
\hline 6 & $\operatorname{Tax}$ & 68.768 .459 & 75.645 .305 & 83.209 .836 & 91.530 .820 & 100.683 .902 & 110.752 .292 & 121.827 .521 & 134.010 .273 \\
\hline 7 & Profit netto & $(8.715 .030)$ & 2.347 .722 & 14.516 .749 & 27.902 .679 & 42.627 .203 & 178.166 .730 & 195.983 .403 & 215.581 .743 \\
\hline
\end{tabular}

Sell price Rp. 2.699,25 /piece

Main price Rp. 2.076,34 /piece

Capacity production $\mathbf{2 8 8 . 0 0 0}$ piece/year

Investation Rp. 320.054.000

\section{Loan Rp. 240.040.500}

Equity loan (Flat) 29,72 \%/year = Rp. 247,69 /piece

Payment Rp. 167 /bh

Taxes 10,0 \% = Rp. 238,78 /piece

Cost production estimation 


\section{ESTIMATION INVESTATION AND COST PRODUCTION TILE CERAMIC}

\section{List Price Unit Tools Production Tile Ceramic}

Capacity : 288.000 pieces/year

\begin{tabular}{|c|c|c|c|c|c|}
\hline \multirow{2}{*}{ No. } & \multirow{2}{*}{ Kind of Tools } & \multirow{2}{*}{ Quantity } & \multirow{2}{*}{ Unity } & \multicolumn{2}{|c|}{ Price $\mathbf{R p}$. } \\
\hline & & & & Unit & Total \\
\hline 1 & $\begin{array}{l}\text { Machine to make tile } \\
\text { ceramic }\end{array}$ & 2 & Unit & $20.000 .000,00$ & $40.000 .000,00$ \\
\hline 2 & Mixer machine & 1 & Unit & $20.000 .000,00$ & $20.000 .000,00$ \\
\hline 3 & Tool assist & 1 & Unit & $5.000 .000,00$ & $5.000 .000,00$ \\
\hline \multicolumn{4}{|r|}{ Total } & \multicolumn{2}{|r|}{$65.000 .000,00$} \\
\hline
\end{tabular}

Total Machines and Tools $=$ Rp. 65.000.000,00

Depreciation per year $=$ Rp. $6.500 .000,00$

Depreciation per piece product $=$ Rp. 22,57

Investation Unit Production Tile Ceramic

Capacity : 288.000 pieces/year

\begin{tabular}{|c|l|r|}
\hline No. & \multicolumn{1}{|c|}{ COST OF INVESTATION } & \multicolumn{1}{c|}{ PRICE } \\
& \multicolumn{1}{|c|}{ (Rp.) } \\
\hline 1 & TOOLS PRODUCTION TILE CERAMIC & $65.000 .000,00$ \\
2 & COST INSTALLATION ELECTRICITY AND WATER & $5.000 .000,00$ \\
3 & LAND FACTORY \& CONCESSION $\left(250 \mathrm{~m}^{2}\right)$ & $125.000 .000,00$ \\
4 & BUILDING PRODUCTION $\left(125 \mathrm{~m}^{2}\right)$ & $125.000 .000,00$ \\
5 & EQUITY WORK 3 MONTHS & $54.000,00$ \\
\hline
\end{tabular}




\section{Source Payment}

Credit bank $=75 \%=\mathbf{2 4 0 . 0 4 0 . 5 0 0 , 0 0}$

Self equity $=25 \%=80.013 .500,00$

T O T A L $\quad=320.054 .000,00$

\section{Estimation Cost Production Tile Ceramic}

Capacity : 288.000 pieces/year

Salary worker

\begin{tabular}{|c|l|c|c|c|}
\hline No. & Explanation & Salary/month (Rp) & Worker & Total \\
\hline $\mathbf{1}$ & Direct Salary & & 3 & $5.100 .000,00$ \\
& Worker & $1.700 .000,00$ & 3 & $4.500 .000,00$ \\
\hline
\end{tabular}

Direct Salary

Salary/month $=$ Rp. 9.600.000,00

Salary/year $=$ Rp. 115.200.000,00

Allowance 1 Month Salary = Rp. 9.600.000,00

Total Salary \& Allowance $=$ Rp. 124.800.000,00

Salary \& Allowance/piece $=$ Rp. 433,33

\section{Estimation Cost Production Tile Ceramic}

Capacity Production : 9.600 pieces/10 days

Mixture composition $1: 3$

Day work : $\mathbf{3 0 0}$ days/year

Capacity per year : $\mathbf{2 8 8 . 0 0 0}$ pieces/year

Direct Cost :

\begin{tabular}{|l|c|r|r|r|}
\hline \multicolumn{1}{|c|}{ Explanation } & Unity & \multicolumn{1}{|c|}{ Volume } & \multicolumn{1}{c|}{$\begin{array}{c}\text { Unit Price } \\
\text { (Rp) }\end{array}$} & \multicolumn{1}{c|}{$\begin{array}{c}\text { Total Price } \\
\text { (Rp) }\end{array}$} \\
& & & & \\
Solar and Olie & ltr & 288 & $6.700,00$ & $1.929 .600,00$ \\
Sand & gr & 136.573 .517 & & $102.430 .137,60$
\end{tabular}




\begin{tabular}{|c|c|c|c|c|}
\hline & & & 0,75 & \\
\hline Clay peat & gr & 497.517 .811 & 0,50 & $248.758 .905,60$ \\
\hline \multirow{2}{*}{$\begin{array}{l}\text { Electricity } \\
\text { Material and energy }\end{array}$} & Kwh & 2.400 & $4.200,00$ & $10.080 .000,00$ \\
\hline & & & & $363.198 .643,20$ \\
\hline Salary & & & & - \\
\hline & & & Total & $363.198 .643,20$ \\
\hline
\end{tabular}

Indirect Cost :

\begin{tabular}{|l|c|c|c|c|}
\hline \multicolumn{1}{|c|}{ Explanation } & Unity & Volume & Unit Price & $\begin{array}{c}\text { Total Price } \\
\text { (Rp) }\end{array}$ \\
Depreciation & $\%$ & 5 & $65.000 .000,00$ & $3.250 .000,00$ \\
Overhead & $\%$ & 5 & & - \\
\hline Maintenance & Ls & 0,05 & $65.000 .000,00$ & $3.250 .000,00$ \\
\hline Salary & & & \\
\hline
\end{tabular}

Payment + Equity Bank per year $=190.677 .004,26$

TOTAL $(1+2+3)=560.375 .647,46$

MAIN PRICE PER PIECE $=1.945,75$

Profit $=20 \%=\mathbf{3 8 9 , 1 5}$

Taxes $=10 \%=\mathbf{1 9 4 , 5 7}$

SELL PRICE PER PIECE $=2.529,47$

Break Event Point (BEP)

Unit Production Tile Ceramic Capacity : $\quad 9.600$ pieces/10 days 288.000 pieces/year 


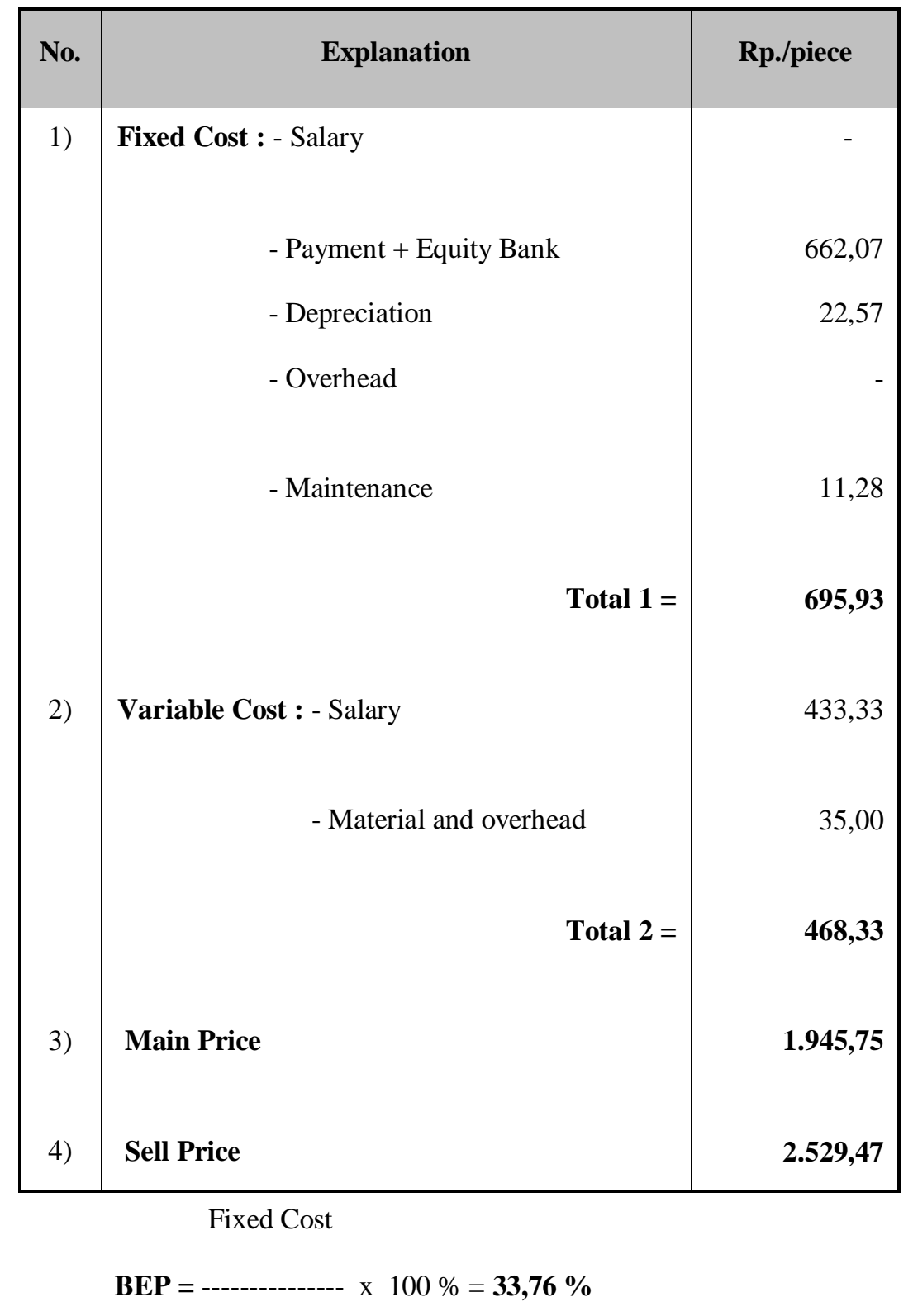

H. jual - Var. Cost 
Cash flow unit production tile ceramic

\begin{tabular}{|c|c|c|c|c|c|c|c|c|c|c|c|}
\hline No. & Explanation & 2016 & 2017 & 2018 & 2019 & 2020 & 2021 & 2022 & 2023 & 2024 & 2025 \\
\hline 1 & Balance cash beginning & 0 & 0 & 168.112 .694 & 353.036 .658 & 556.453 .018 & 780.211 .014 & 1.026 .344 .810 & 1.297.091.985 & 1.594 .913 .878 & 1.922 .517 .959 \\
\hline 2 & Income & 320.054 .000 & 728.488 .342 & 801.337 .176 & 881.470 .893 & 969.617 .983 & 1.066 .579 .781 & 1.173.237.759 & 1.290 .561 .535 & 1.419 .617 .689 & 1.561.579.457 \\
\hline 3 & Outcome & 320.054 .000 & 560.375 .647 & 616.413 .212 & 678.054 .533 & 745.859 .987 & 820.445 .985 & 902.490 .584 & 992.739 .642 & 1.092.013.607 & 1.201.214.967 \\
\hline 4 & Difference cash (2-3) & 0 & 168.112 .694 & 184.923 .964 & 203.416 .360 & 223.757 .996 & 246.133 .796 & 270.747 .175 & 297.821 .893 & 327.604 .082 & 360.364 .490 \\
\hline 5 & Balance cash ending & 0 & 168.112 .694 & 353.036 .658 & 556.453 .018 & 780.211 .014 & 1.026 .344 .810 & 1.297 .091 .985 & 1.594.913.878 & 1.922.517.959 & 2.282 .882 .450 \\
\hline
\end{tabular}

Main price red brick $=$ Rp. 1.945,75 $/$ piece

Sell price red brick $=$ Rp. 2.529,47 /piece

Capacity production $=\mathbf{2 8 8 . 0 0 0}$ pieces/year

Cost production tile ceramic estimation

increase as much as =:10 \%/year

Total investation $=$ Rp. 320.054.000,- 


\section{Calculation Internal Rate of Return (IRR) Production Tile Ceramic}

Capacity Prod.: 288.000 pieces/year

Sell Price : 2.529,47 rupiah/piece

Main Price : 1.945,75 rupiah/piece

Investation : 320.054.000,00 rupiah

\begin{tabular}{|c|c|c|c|c|c|c|}
\hline Year & $\mathbf{0}$ & 1 & 2 & 3 & 4 & 5 \\
\hline Income & 0 & 728488342 & 728488341,7 & 728488342 & 728488342 & 728488341,7 \\
\hline Outcome & 320054000 & 560375647 & 560375647,5 & 560375647 & 560375647 & 560375647,5 \\
\hline Net Cash Flow : & -320054000 & 168112694 & 168112694,2 & 168112694 & 168112694 & 168112694,2 \\
\hline NPV $50 \%$ & 1 & 0,6666667 & 0,444444444 & 0,2962963 & 0,19753086 & 0,131687243 \\
\hline NPV $40 \%$ & 1 & 0,7142857 & 0,510204082 & 0,36443149 & 0,2603082 & 0,185934432 \\
\hline NPV $30 \%$ & 1 & 0,7692308 & 0,591715976 & 0,45516614 & 0,3501278 & 0,269329074 \\
\hline NPV $20 \%$ & 1 & 0,8333333 & 0,694444444 & 0,5787037 & 0,48225309 & 0,401877572 \\
\hline
\end{tabular}

Total NPV $50 \%:=\mathbf{- 2 8 1 0 5 2 0 5 , 8 9}$ Rupiah

Total NPV $40 \%:=\mathbf{2 2 . 0 8 2 . 8 8 9 , 7 7}$ rupiah

Total NPV $30 \%:=\mathbf{8 9 . 3 9 6 . 1 9 3 , 0 4}$ rupiah

Total NPV $20 \%:=\mathbf{1 8 2 . 7 0 5 . 8 6 4 , 2 6}$ rupiah

$\mathrm{IRR}=\mathbf{4 3 , 2 8} \%$

Pay Back Period = 1,90 year

Profit $=\mathbf{2 0 , 0 0} \%$ 
Balance Sheet Loss and Profit Production Tile Ceramic

\begin{tabular}{|c|c|c|c|c|c|c|c|c|c|c|}
\hline No. & Explanation & 2016 & 2017 & 2018 & 2019 & 2020 & 2021 & 2022 & 2023 & 2024 \\
\hline 1 & Income & 728.488 .342 & 801.337 .176 & 881.470 .893 & 969.617 .983 & 1.066 .579 .781 & 1.173 .237 .759 & 1.290 .561 .535 & 1.419 .617 .689 & 1.561 .579 .457 \\
\hline 2 & Cost Production & 560.375 .647 & 616.413 .212 & 678.054 .533 & 745.859 .987 & 820.445 .985 & 902.490 .584 & 992.739 .642 & 1.092 .013 .607 & 1.201 .214 .967 \\
\hline 3 & $\begin{array}{l}\text { Profit before equity \& } \\
\operatorname{tax}\end{array}$ & 168.112 .694 & 184.923 .964 & 203.416 .360 & 223.757 .996 & 246.133 .796 & 270.747 .175 & 297.821 .893 & 327.604 .082 & 360.364 .490 \\
\hline 4 & Equity loan + payment & 119.342 .552 & 119.342 .552 & 119.342 .552 & 119.342 .552 & 119.342 .552 & 0 & 0 & 0 & 0 \\
\hline 5 & Profit before tax & 48.770 .142 & 65.581 .412 & 84.073 .808 & 104.415 .444 & 126.791 .244 & 270.747 .175 & 297.821 .893 & 327.604 .082 & 360.364 .490 \\
\hline 6 & $\operatorname{Tax}$ & 64.443 .199 & 70.887 .519 & 77.976 .271 & 85.773 .898 & 94.351 .288 & 103.786 .417 & 114.165 .059 & 125.581 .565 & 138.139 .721 \\
\hline 7 & Profit netto & $(15.673 .057)$ & (5.306.108) & 6.097 .537 & 18.641 .545 & 32.439 .955 & 166.960 .758 & 183.656 .834 & 202.022 .517 & 222.224 .769 \\
\hline
\end{tabular}

Sell price $=$ Rp. $\mathbf{2 . 5 2 9 , 4 7 ~} /$ piece

Main price $=$ Rp. 1.945,75 $/$ piece

Capacity production $=\mathbf{2 8 8 . 0 0 0}$ piece/year

Investation $=$ Rp. 320.054.000

Loan $=$ Rp. 240.040.500

Equity loan $($ Flat $)=\mathbf{2 9 , 7 2} \% /$ year $=$ Rp. 247,69 $/$ piece

Payment $=$ Rp. $167 / b h$

Taxes $=\mathbf{1 0 , 0} \%=$ Rp. 223,76 $/$ piece

Cost production estimation increase as much as $=\mathbf{1 0} \%$ every year 
Price red brick in market $=$ Rp. 600,- per piece, while while price red brick production result $=\mathrm{Rp}$. 2.700,- per piece. Price red brick production result more expensive, cause of calculation feasibility study using analyze making industry red brick, while add buying machine, buying land production, making building production, land concession for road to location production, cost of depreciation, profit, taxes, bank equity, installation electricity and water, working capital for 3 months, worker salary, and supported material.

Red brick production result have better quality, compared with red brick that sold in market, cause red brick production result using mixture composition 1 sand : 3 soil peat, while red brick that sold in market using mixture composition 1 sand : 5 clay until 1 sand : 6 clay. That matter is same with quality tile ceramic, have better quality if compared with tile ceramic that sold in market.

Price tile ceramic in market $=$ Rp. 9.700,- more expensive from price tile ceramic that produced by production $=$ Rp. 2,550,-. This is caused tile ceramic that sold in market have layered by glazing, and processed burning in oven. But tile ceramic production result also have burned in furnace, until $900{ }^{\circ} \mathrm{C}$.

\section{CONCLUSION}

In make one house type $49 \mathrm{~m}^{2}$, need $\pm 7,875 \mathrm{red}$ brick and $\pm 3,675$ tile ceramic. Or 7,875 x Rp. $2.700,-+3,675$ x Rp. $2,550,-=$ Rp. $30,633,750,-$

Price one house type $49 \mathrm{~m}^{2}=$ Rp. 30,633,750,-, only from price wall from red brick and price roof from tile ceramic.

\section{REFERENCES}

[Google.com], 2009, Utilization and management soil peat in Central Kalimantan, Date of access: 09.09.2015.

[Final Report], RANDING, ET ALL, 1998, Development building material in soil peat Central Kalimantan, Research Institute for Human Settlements, Public Work Ministry.

[Proceedings, HabiTechno International Seminar, Public Housing \& Technological Innovations For Sustainable Urbanization], AVENTI, 2015, Soil peat of Central Kalimantan as local building material, School of Architecture, Planning, and Policy Development, Institut Teknologi Bandung, Indonesia.

[Google.com], 2008, That disaster Project development peat land 1 million hectare, project peat land 1 million hectare. Date of access: 09.09.2015. 